\title{
Nanoscale
}

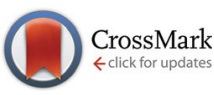

Cite this: Nanoscale, 2016, 8, 10922

Received 25th April 2016,

Accepted 3rd May 2016

DOI: $10.1039 / \mathrm{c} 6 \mathrm{nr} 03349 \mathrm{k}$

www.rsc.org/nanoscale

\section{A biologically inspired attachable, self-standing nanofibrous membrane for versatile use in oil-water separation $\uparrow$}

\author{
Mizuki Tenjimbayashi, Kaichi Sasaki, Takeshi Matsubayashi, Jyunichiro Abe, \\ Kengo Manabe, Sachiko Nishioka and Seimei Shiratori*
}

\begin{abstract}
Uloborus walckenaerius spider webs provided the inspiration for attachable, self-standing nanofibre sheets. The developed product adds selective wettability against oil-water mixtures to both 2D and 3D materials by attaching or covering them, leading to successful separation through a facile, scalable and low-cost process.
\end{abstract}

Oil pollution in water is a worldwide problem, causing longterm effects on the health of living organisms and the environment. ${ }^{1,2}$ However, high-purity oil is an important energy resource on which humans depend to maintain their current lifestyle. ${ }^{3}$ The refuse disposal systems currently available for industrial effluents from factories or for burning rubbish are both expensive and time-consuming. ${ }^{4}$ It is therefore important to develop methods for the extraction of oil and other chemical pollutants and to remove pure oil from water. ${ }^{5}$

Recent approaches to oil-water separation are based on the selective wetting of oils, chemicals and water onto $2 \mathrm{D}$ or $3 \mathrm{D}$ porous materials. ${ }^{6}$ The techniques for oil-water separation include: (i) modifying nano- and microporous 2D structures with alkyl- or fluoroalkyl-terminated compounds to design (super)hydrophobic and (super)oleophilic porous sheets; ${ }^{7,8}$ (ii) modifying nano- and microporous structures with compounds containing - $\mathrm{OH}$ groups to make superhydrophilic and superoleophobic surfaces under water or hygro-responsive conditions; ${ }^{9-11}$ (iii) modifying natural 3D porous structures with compounds containing alkyl chains; ${ }^{12,13}$ (iv) synthesizing 3D-structured materials via freeze-drying methods using a cross-linked hydrophobic polymer to design (super)hydrophobic and oil-absorbable 3D materials $;^{14,15}$ and (v) designing porous surfaces with an omniphobic lubricant to open pores triggered by variations in pressure to selectively pass liquids

School of Integrated Design Engineering, Keio University, 3-14-1 Hiyoshi, Yokohama, 223-8522, Japan.E-mail: shiratori@appi.keio.ac.jp

$\dagger$ Electronic supplementary information (ESI) available: Experimental section, designing procedure, cost, cross sectional SEM, influence of NFs-S components to wettability, thickness, fibre diameter and flexibility, surface tension $v s$. contact angle, SEM images after extraction of oil, characteristics of testing oil, large scale-fabrication of NFs-S. See DOI: 10.1039/c6nr03349k through the membrane (inspired by the gating mechanism of plant stoma). ${ }^{16}$

In these approaches, the materials designed for oil-water separation need to make use of both $2 \mathrm{D}$ and $3 \mathrm{D}$ structured porous solids with a specific supporting body. This technology has already achieved the highly effective and continuous separation of oil from mixtures and emulsions using strategies of oil-water filtration, ${ }^{17,18}$ oil absorption ${ }^{12,19,20}$ and oil collection. ${ }^{21-23}$ The challenges of oil-water separation are: (i) adding other functions, such as transparency, ${ }^{18}$ durability, ${ }^{23}$ self-healing ${ }^{24}$ and a stimuli-response; ${ }^{10,25}$ (ii) working under special conditions at different pressures ${ }^{26}$ or under ambient conditions at different temperatures, ${ }^{13}$ or in the presence of other chemicals; ${ }^{1}$ and (iii) making this technology scalable, facile and low cost. ${ }^{6,11}$ The last requirement, in particular, is essential in practical applications. Hence our aim was to develop a scalable, facile and low-cost material that could be applied to both 2D and 3D structured porous solids for various wetting-controlled oil-water separation techniques.

Our strategy was to design a self-standing sheet for oilwater separation that could be attached to various materials to provide this function to both $2 \mathrm{D}$ and $3 \mathrm{D}$ materials. The conventional approach to this requires chemical bonding or physical cross-linking between the supporting material and the functional material; however, this type of versatile attachment is difficult to design.

Nature offers a remarkably simple alternative idea. The spider Uloborus walckenaerius weaves an electrically charged thread into fluffed nanofibres from individual spigots using specialized combs on its legs. The webs capture flying insects by electrostatic van der Waals forces (Fig. 1A). ${ }^{27,28}$ Inspired by this capture mechanism, ${ }^{29}$ we designed a randomly aligned nanofibre sheet (NF-S) using the easily chargeable fluorine polymer of poly(vinylidene fluoride-co-hexafluoropropylene) (PVDF-HFP) (Fig. 1B). Electrostatic van der Waals forces enabled the NF-S to attach to various materials, such as a Kimtowel and a finger (Fig. 1C-E). ${ }^{30}$

The NF-S was fabricated by an electrospinning method, resulting in the facile design of various oil-water separating 

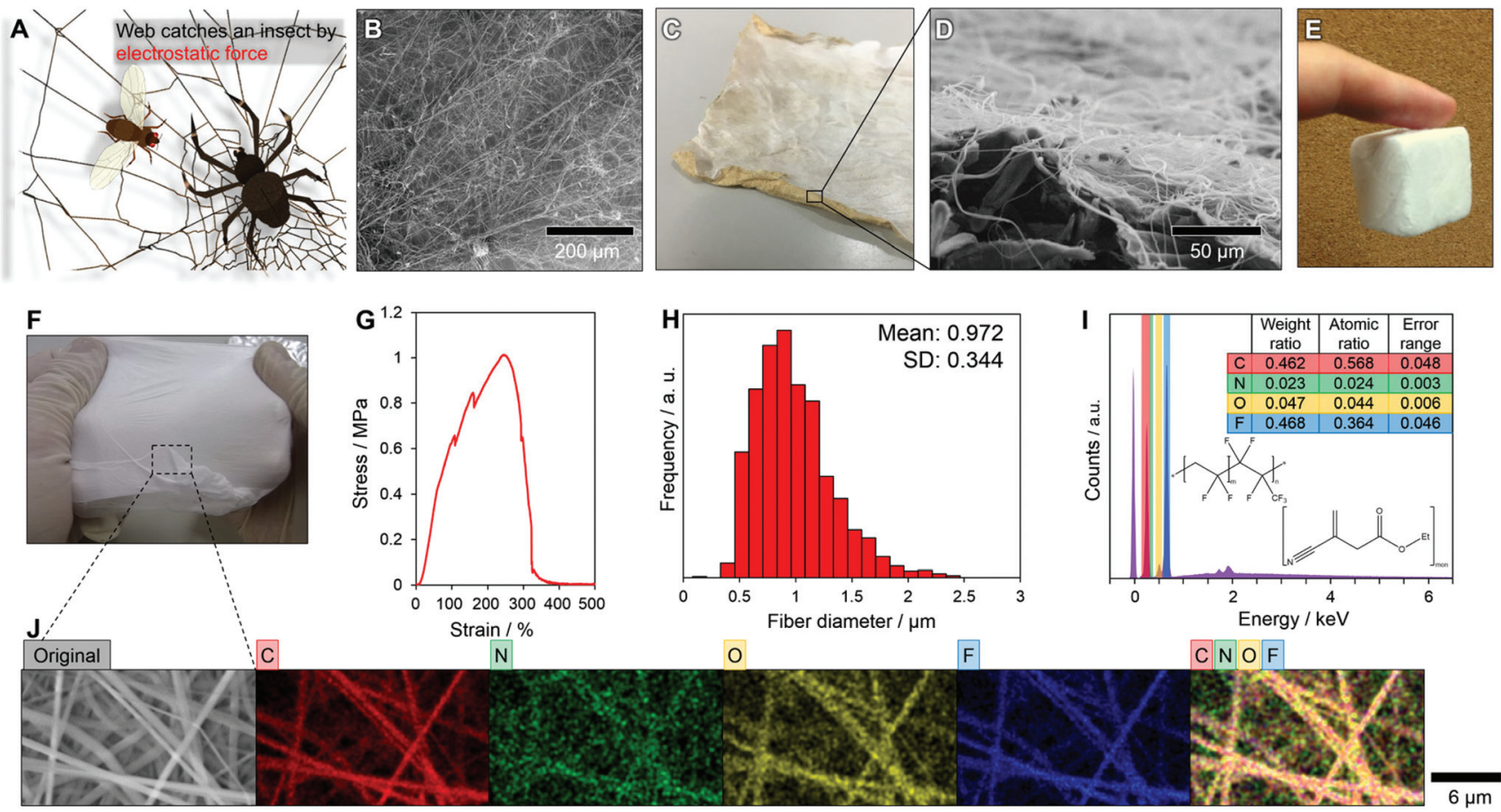

$\mathrm{N}$
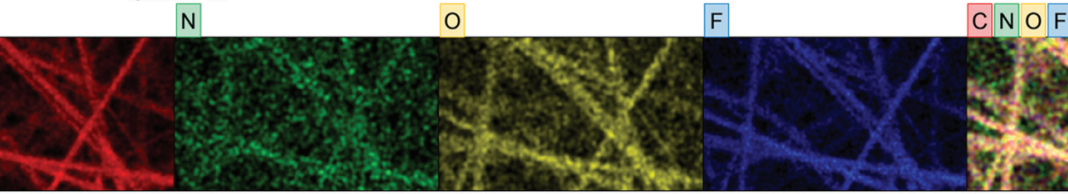

Fig. 1 Biomimicry of Uloborus walckenaerius spider web to fabricate an attachable NF-S. (A) Schematic diagram of the predation mechanism of U. walckenaerius. (B) SEM image of an attachable NF-S. (C) Attachment of an NF-S to a Kimtowel by electrostatic van der Waals forces. (D) Magnified image of (C) (E) attachment of a NF-S block to a finger by electrostatic van der Waals forces. (F-J) Characteristics of the self-standing NF-S. (F) Photograph of the self-standing NF-S. (G) NF-S strength and elastic properties. (H) Distribution of fibre diameters in the NF-S. (I) Elemental composition of NF-S. (J) Elemental distribution of carbon, nitrogen, oxygen, fluorine and overall distribution.

devices (Fig. S1†). The NF-S, with adjustable wettability, strength and elastic properties provided by ethyl-alpha-cyanoacrylate (Et-CA), was successful in oil-water separation with both a 2D structure using a self-standing NF-S and a 3D structure by attaching an NF-S. Indeed, a self-standing property of NF-S is shown in Fig. 1F. It may also be possible to design an advanced oil-water separator by selecting a functional material as the supporting body for the NF-S. The new NF-S could have oil-water separation. The cost of the materials used to fabricate the NF-S reported here (PVDF-HFP, Et-CA and acetone) was about US\$ $0.88 \mathrm{~m}^{-2}$ (Table S1 $\dagger$ ). The NF-S is self-standing and stretchable. The maximum strain of the NF-S was about $250 \%$ at a stress $>1 \mathrm{MPa}$ (Fig. 1G). The fracture behaviour of the NF-S in a strain-stress test was gradual, meaning that the thinner fibres within the sheet fractured first, then the thicker fibres, resulting in a gradual decrease in the stress at strains $>250 \%$. The distribution of the fibre diameters within the NF-S is shown in Fig. $1 \mathrm{H}$; these ranged from 0.3 to $2.5 \mu \mathrm{m}$ with a mean diameter of $0.972 \pm 0.344 \mu \mathrm{m}$. Thus a mixture of microfibres and nanofibres provided the strength of the NF-S. The thickness of the NF-S was about $135 \mu \mathrm{m}$ (Fig. S2 $\uparrow$ shows a cross-sectional scanning electron microscopy image of the NF-S). The surface chemistry of the fibres was analysed by energy-dispersive X-ray spectrosmetry (EDX) (Fig. 1I and J). The NF-S was composed of PVDF-HFP (hydrogen, carbon and fluorine) and Et-CA (hydrogen, carbon, nitrogen and oxygen). The EDX mapping (Fig. 1I) showed carbon, nitrogen, oxygen and fluorine peaks. The exist- ence of fluorine indicates the presence of PVDF-HFP. The atomic ratio of nitrogen to oxygen was about $1: 2$, which means that the fibres included Et-CA. The EDX analysis confirmed that each element was included within the fibres (Fig. 1J). The strength, strain, fibre diameter and wettability of the NF-S was controlled by changing the weight ratio of PVDF-HFP to Et-CA (Table S2 and Fig. S3-S8†).

The NF-S is characterized by a selective wettability for oilwater separation. We studied the water blocking and oil penetration mechanisms for the NF-S in detail (Fig. 2). The contact angles of water and oils on the NF-S surface are shown in Fig. $2 \mathrm{~A}$ and $\mathrm{B}$, respectively. The water blocking ability of the NF-S was tested for the bare NF-S (Fig. 2Ai), toluene-infused NF-S (Fig. 2Aii) and hexadecane-infused NF-S (Fig. 2Aiii). The water droplets did not penetrate the surface under any of these conditions. Fig. 2Biv and $\mathrm{v}$ show that the oil droplets (toluene and hexadecane, respectively) penetrated the bare NF-S. Fig. 2C shows time-lapsed images for each of these examples (i)-(v). In example (i) (Fig. 2D), the NF-S showed the highest hydrophobic state towards the water droplet, which did not penetrate the surface. In examples (ii) and (iii), we found that an oily layer formed on the NF-S surface and blocked water invasion (as a result of the immiscibility of oil and water) (Fig. 2E, example (iii)). ${ }^{31}$ Fig. $2 \mathrm{~F}$ shows an image of an oil droplet that penetrated the NF-S. Some of the oil remained on the surface of the NF-S, but most of the oil penetrated the NF-S, which allowed oil-water separation. The oil layer formed 
A
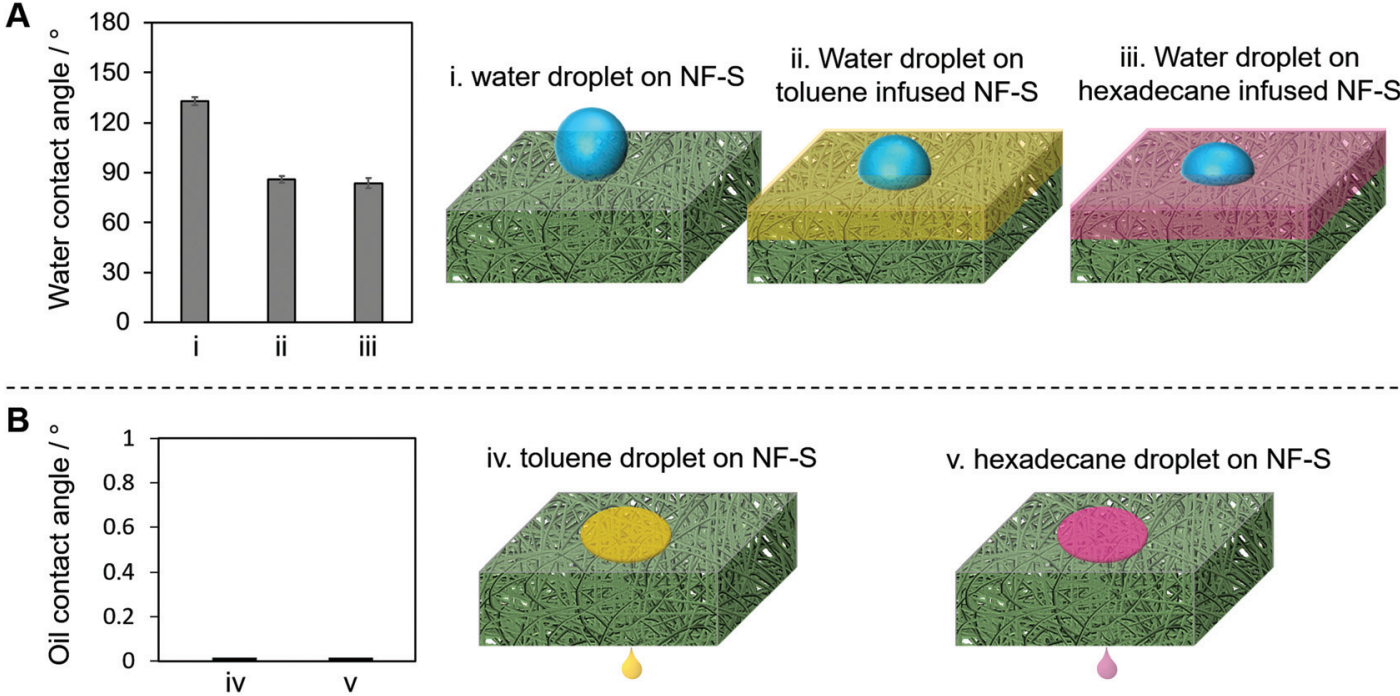

iv. toluene droplet on NF-S

v. hexadecane droplet on NF-S
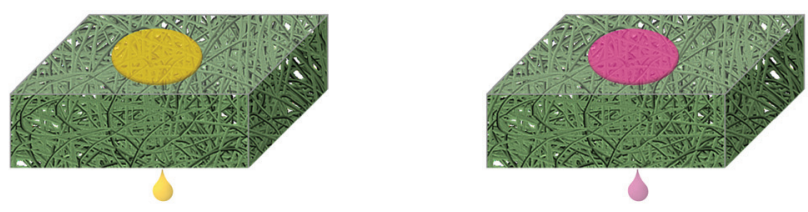

C
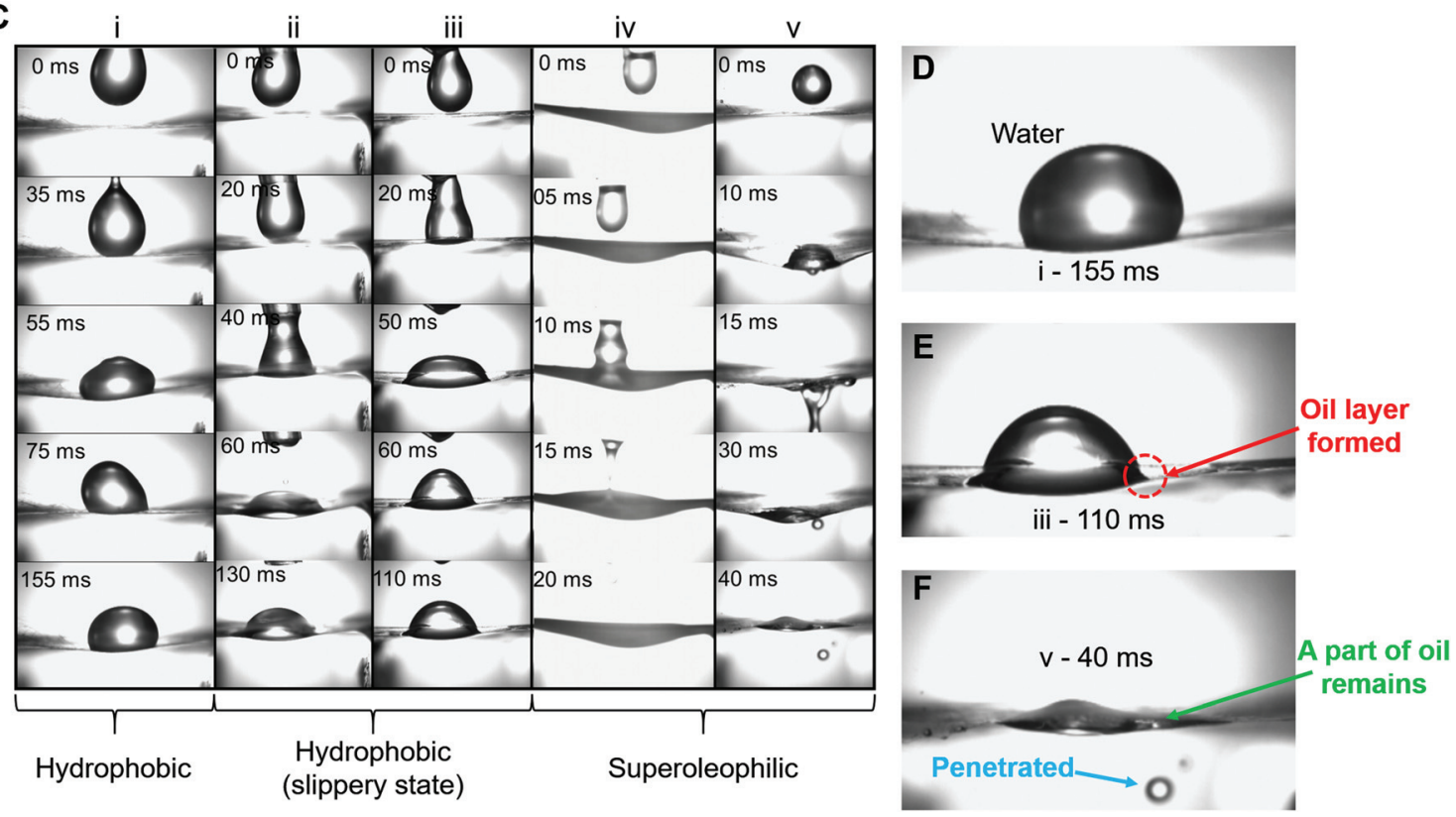

Fig. 2 Wetting behaviour of water and oils on the NF-S showing the mechanism of oil-water separation. (A) Water contact angles and schematic illustrations of a water droplet on: (i) the NF-S; (ii) a toluene-infused NF-S; and (iii) hexadecane-infused NF-S. (B) Oil contact angles and schematic illustrations of (iv) a toluene droplet and (v) a hexadecane droplet on the NF-S. (C-F) Hydrophobicity and oil penetration of the NF-S demonstrated by (C) time-resolved images (i)-(v) and(D-F) selected magnified images.

because of the capillary action from the high surface area of the NF-S $\left(1.12 \mathrm{~m}^{2} \mathrm{~g}^{-1}\right.$ determined by the Brunauer-EmmettTeller method). This type of oil layer can block water; the mechanism is similar to that used to design a micro-sponge surface that retains oily liquids (SLIPS), which allows water to slide because of the immiscibility of water with oil. ${ }^{32,33}$ We found that the limit of wetting or repelling was a surface tension of $36.09-38.56 \mathrm{mN} \mathrm{m}^{-1}$ (Fig. S9†).

We applied the NF-S to three types of applications: oil absorption, oil-water separation and oil collection. Selective wetting is well known in oil-water separation using each of these methods. Fig. 3 shows the application of oil absorption.
A melamine sponge was covered with a NF-S to create a 3Dstructured separator (Fig. 3A). Although the sponge was naturally omniphilic, the sponge + NF-S showed hydrophobicity (Fig. 3B) and superoleophilicity. The oil absorbent was used in processes (i)-(iii) in Fig. 3C (Movie S1†). The merit of the combination of sponge + NF-S is that the absorbed oil can be easily extracted for re-use, whereas other oil absorption methods require more complex extraction processes such as heating. ${ }^{12,20}$ This feature comes from the flexibility of the NF-S. The NF-S maintained its structure after oil extraction (Fig. S10 $\dagger$ ) to realize repeatable oil absorption and extraction without deterioration (Fig. 3D) with a high absorption capacity 

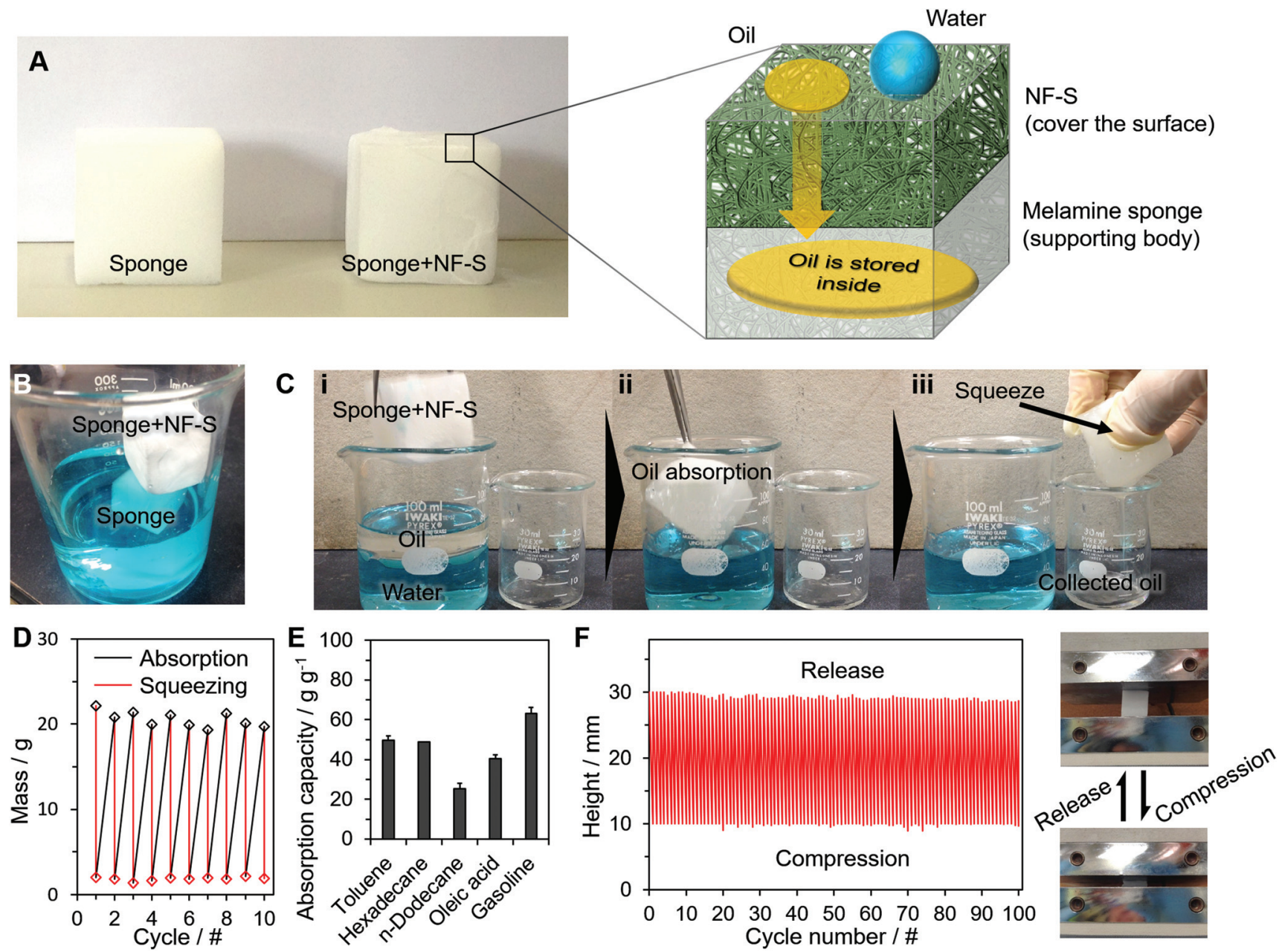

Fig. 3 Application of a sponge + NF-S for 3D oil-water separation by oil absorption. (A) Photograph of an uncoated melamine sponge (sponge) and NF-S attached melamine sponge (sponge + NF-S) with a schematic image of the magnified oil absorbents. (B) Photograph of the samples in a beaker half-filled with water (blue). (C) Photographs of the sponge + NF-S for use in separating oil by (i) placement in a toluene-water mixture, (ii) the sponge + NF-S absorbs the toluene but not water and (iii) squeezing to extract the pure toluene. (D) Mass change of toluene inside the sponge with cycles of absorption and squeezing. (E) Absorption capacities for the oil-water mixtures with different types of oil. (F) Cyclical pressing measurements for the sponge + NF-S.

for various types of oil (Fig. 3E). We also conducted a compression test to determine the 3D structural retention, which showed the high stability of the sponge structures (Fig. 3F). These functions were derived from the natural properties of the melamine sponge. Thus we added a selective wetting property to the sponge, which already had stable liquid storage properties and high compression durability. This is an example of an addition of function to an already functional material to create an advanced material.

Fig. 4 shows the application of the material in an oil-water filter. The NF-S was attached to the tip of a PVC tube and the oil-water separation is shown in Fig. 4A (i)-(iii) (Movie S2†). When an oil-water mixture was poured into the PVC tube, only oil penetrated the NF-S and was collected in a container below the PVC tube (Fig. 4B). The purity of the separation was determined by changing the type of oil and gave an average separation efficiency of $>99 \%$ (Fig. 4C). The separation efficiencies varied slightly based on the oil retained on the NF-S. The flux value for each type of oil is shown in Fig. 4D. The flux $(f)$ is related to the viscosity of the oil $(\mu)$ by $f=\mu d g L^{-1}$, where $L$ and $d$ are the thickness and pore diameter of the NF-S, respectively, and $g$ is the gravitational constant ${ }^{34}$ (Table S3 $\dagger$ ). Thus the separation flux decreased as the thickness increased (Fig. 4E) and the pore size decreased (Fig. 4F). The flux for gasoline permeating the membrane was $c .3000 \mathrm{~L} \mathrm{~m}^{-2} \mathrm{~h}^{-1}$.

We compared the separation properties for our NF-S with those of other reported filter membranes (Table S4†). Although the separation efficiency for our NF-S was similar to that for other membranes, the cost to produce the NF-S was much lower than most of the other membranes. The system was also used as an oil collector (Fig. 4E) ( $(\mathrm{S} 3 \dagger)$. The container covered with the NF-S allowed oil penetration so, after pouring an oilwater mixture through the material, only oil was collected in the container. The NF-S (folded five times) was also used to 
A

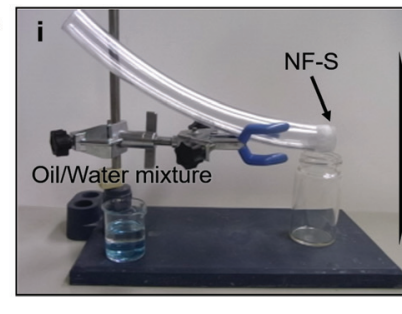

C

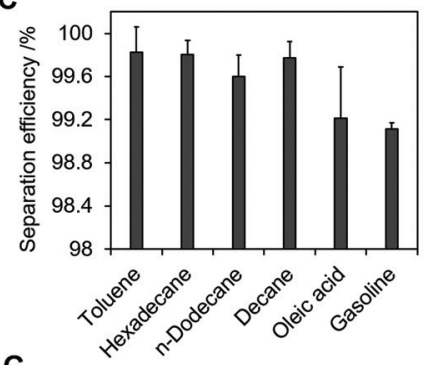

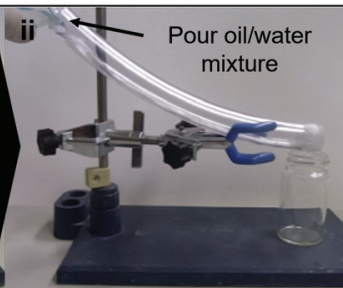

D

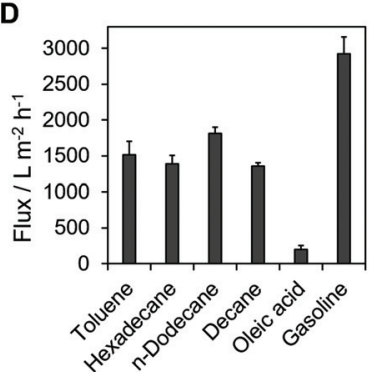

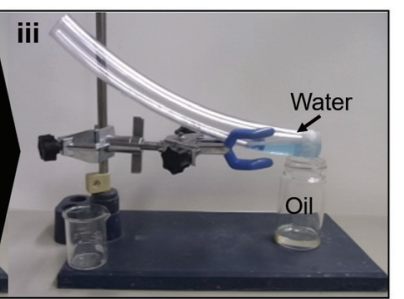

E

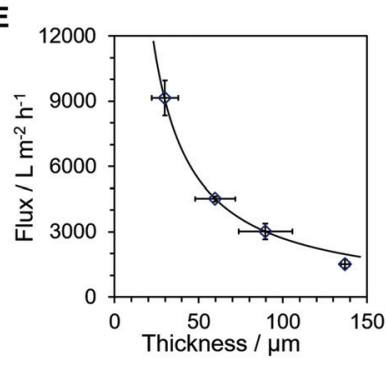

B

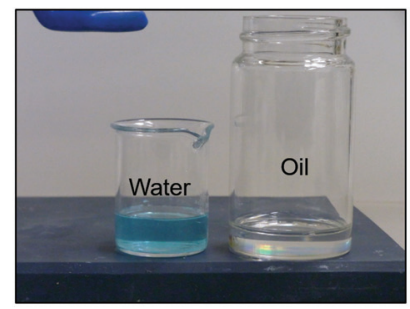

$\mathbf{F}$

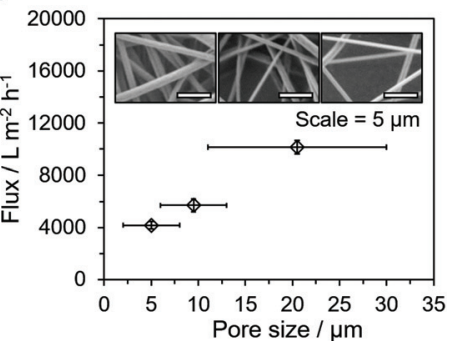

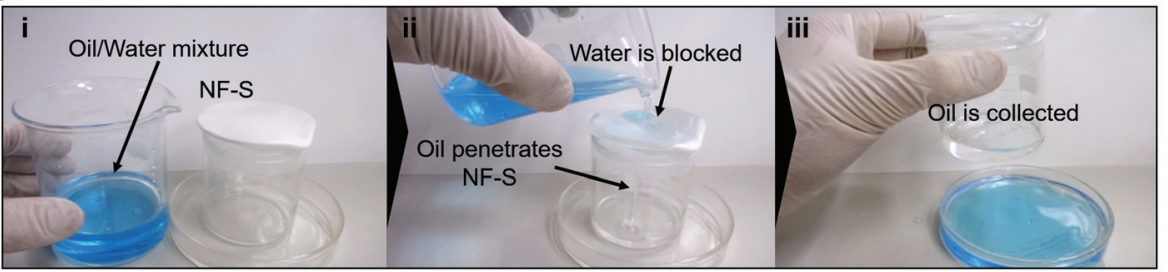
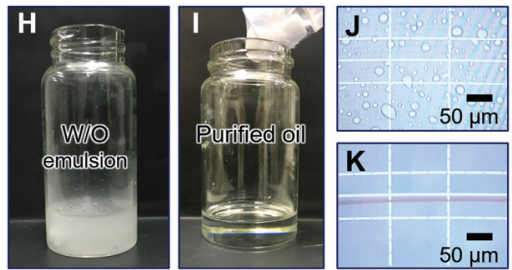

Fig. 4 Application of 2D oil-water separation. (A) Oil-water separation filter. Photographs show the separation of toluene from the toluene-water mixture in steps (i)-(iii). (B) Photograph of toluene and water after separation. (C) Separation efficiencies, and (D) separation flux of oil-water mixtures with different types of oil. (E, F). Separation flux of the oil-water mixtures with different NF-S thicknesses (E) and pore diameters (F) adjusted by folding and extending the NF-S, respectively. (G) Application of an oil collector. Photograph of toluene collection in a container from a toluenewater mixture in processes (i)-(iii). ( $\mathrm{H}-\mathrm{K})$ Photographs before $(\mathrm{H}, \mathrm{J})$ and after $(\mathrm{I}, \mathrm{K})$ emulsion.

extract pure oils from an emulsified oil-water mixture without assistance from pressure (Fig. $4 \mathrm{H}-\mathrm{K}$ ) and provided an effective separation.

We designed a large-scale electrospinning device for the large-scale fabrication of the NF-S using a combination of a slider and drum roll (Fig. S11 $\dagger$ ) to manufacture a NF-S at a size of $600 \times 500 \mathrm{~mm}$. This increases the number of possibilities for the types of $2 \mathrm{D}$ and $3 \mathrm{D}$ materials that can be created for oil-water separation.

\section{Conclusions}

We designed a self-standing, super-elastic and adhesive NF-S with anti-wetting/super-wetting properties towards water and oily liquids, inspired by the $U$. walckenaerius spider web. The NF-S was used in three types of oil-water separations: 3D oil absorption; 2D oil-water filtration; and oil collection. The sheet offers an easy, low-cost and scalable process for effective oil-water separation and the adhesive nature of the sheet (through van der Waals interactions), which is independent of the supporting body, offers the possibility of designing advanced materials by combining different functional materials.

\section{Author contribution}

M. Tenjimbayashi designed the experiments, devised the explanation, and wrote the manuscript. M. Tenjimbayashi, K. Sasaki, S. Nishioka collected the data. M. Tenjimbayashi, T. Matsubayashi, J. Abe and K. Manabe analysed the data. All authors discussed the results and reviewed the manuscript.

\section{Acknowledgements}

We are grateful to Dr Kouji Fujimoto, Dr Kyu-Hong Kyung, Dr Yoshio Hotta, Mr Masatsugu Komine, Mr Takeo Moriya, Mr Kouki Kawase and Mr Taichi Nakashima, whose meticulous comments were of enormous help. We would like to thank Prof. Walter Navarrini in Politecnico di Milano for technical support. A part of this work is supported by Grant-in-Aid for Scientific Research -KAKENHI (Grant number: 16J06070).

\section{References}

1 L. Wang, Y. Zhao, Y. Tian and L. Jiang, Angew. Chem., Int. Ed., 2015, 54, 14732. 
2 X. Liu, R. Meng, Q. Xing, M. Lou, H. Chao and L. Bing, Ocean Coast. Manag., 2015, 108, 140.

3 K. Jayaramulu, K. Kumara, R. Datta, C. Rçsler, M. Petr, M. Otyepka, R. Zboril and R. A. Fischer, Angew. Chem., Int. Ed., 2016, 55, 1178.

4 R. O. Crist and R. A. R. Boaventura, J. Cleaner Prod., 2015, 87, 603.

5 G. Kwon, E. Post and A. Tuteja, MRS Commun., 2015, 5, 475.

6 Z. Chu, Y. Feng and S. Seeger, Angew. Chem., Int. Ed., 2015, 54, 2328.

7 W. Zhang, N. Liu, Y. Cao, Y. Chen, L. Xu, X. Lin and L. Feng, Adv. Mater., 2015, 27, 7349.

8 K. He, H. Duan, G. Chen, X. Liu, W. Yang and D. Wang, ACS Nano, 2015, 9, 9188.

9 A. K. Kota, G. Kwon, W. Choi, J. M. Mabry and A. Tuteja, Nat. Commun., 2012, 3, 1025.

10 J. Li, Y. Zhou and Z. Luo, ACS Appl. Mater. Interfaces, 2015, 7, 19643.

11 G. Wang, Y. He, H. Wang, L. Zhang, Q. Yu, S. Peng, X. Wu, T. Ren, Z. Zeng and Q. Xue, Green Chem., 2015, 17, 3093.

12 Y. Yang, H. Yi and C. Wang, ACS Sustainable Chem. Eng., 2015, 3, 3012.

13 J. Li, L. Shi, Y. Chen, Y. Zhang, Z. Guo, B. Su and W. Liu, J. Mater. Chem., 2012, 22, 9774.

14 J. P. Chaudhary, N. Vadodariya, S. K. Nataraj and R. Meena, ACS Appl. Mater. Interfaces, 2015, 7, 24957.

15 Y. Si, J. Yu, X. Tang, J. Ge and B. Ding, Nat. Commun., 2014, 5,5802 .

16 X. Hou, Y. Hu, A. Grinthal, M. Khan and J. Aizenberg, Nature, 2015, 519, 70.

17 G. Wang, Z. Zeng, H. Wang, L. Zhang, X. Sun, Y. He, L. Li, X. Wu, T. Ren and Q. Xue, ACS Appl. Mater. Interfaces, 2015, 7, 26184.
18 N. Yokoi, K. Manabe, M. Tenjimbayashi and S. Shiratori, ACS Appl. Mater. Interfaces, 2015, 7, 4809.

19 R. Du, X. Gao, Q. Feng, Q. Zhao, P. Li, S. Deng and L. Shi, Adv. Mater., 2015, 201504542.

20 Y. Yang, Y. Deng, Z. Tong and C. Wang, ACS Sustainable Chem. Eng., 2014, 2, 1729.

21 J. Song, Y. Lu, J. Luo, S. Huang, L. Wang, W. Xu and I. P. Parkin, Adv. Mater. Interfaces, 2015, 2, 1500350.

22 P. S. Brown and B. Bhushan, Sci. Rep., 2015, 5, 8701.

23 X. Zhou, Z. Zhang, X. Xu, F. Guo, X. Zhu, X. Men and B. Ge, ACS Appl. Mater. Interfaces, 2013, 5, 7208.

24 W. Fang, L. Liu, T. Li, Z. Dang, C. Qiao, J. Xu and Y. Wang, Chem. - Eur. J., 2016, 22, 878.

25 H. Wang, H. Zhou, W. Yang, Y. Zhao, J. Fang and T. Lin, ACS Appl. Mater. Interfaces, 2015, 7, 22874.

26 X. Gao, L. Xu, Z. Xue, L. Feng, J. Peng, Y. Wen, S. Wang and X. Zhang, Adv. Mater., 2014, 26, 1771.

27 K. Kronenberger and F. Vollrath, Biol. Lett., 2015, 11, 20140813.

28 H. M. Peters, Zoomorphology, 1984, 104, 96.

29 K. Ariga, K. Kawakami, M. Ebara, Y. Kotsuchibashi, Q. Ji and J. P. Hil, New J. Chem., 2014, 38, 5149.

30 R. A. Distasio, O. A. Von Lilienfeld and A. Tkatchenko, Proc. Natl. Acad. Sci. U. S. A., 2012, 109, 14791.

31 T.-S. Wong, S. H. Kang, S. K. Y. Tang, E. J. Smythe, B. D. Hatton, A. Grinthal and J. Aizenberg, Nature, 2011, 477, 443.

32 J. D. Smith, R. Dhiman, S. Anand, E. Reza-Garduno, R. E. Cohen, G. H. McKinley and K. K. Varanasi, Soft Matter, 2013, 9, 1723.

33 M. Cao, D. Guo, C. Yu, K. Li, M. Liu and L. Jiang, ACS Appl. Mater. Interfaces, 2016, 8, 3615-3623.

34 L. Wang, Y. Zhao, Y. Tian and L. Jiang, Angew. Chem., Int. Ed., 2015, 54, 14732. 